\title{
COMMENTARY
}

\section{Research on HIV and breastfeeding: definitions can make all the difference}

\author{
T Greiner
}

International Nutrition Research Group, Department of Women's and Children's Health, Uppsala University Academic Hospital, Uppsala, Sweden

Perhaps all fields feel under-appreciated and underfunded. But, somehow, breastfeeding seems to fall between more than the usual number of cracks. Paediatricians rarely receive more than cursory training in the field. Indeed, even at my own "breastfeedingenlightened" department, eyebrows were initially raised when plans for a full-time 5-wk course on paediatrics were first raised-until the faculty saw the enormous list of topics that needed to be covered.

Outside of the field of paediatrics (with the possible exception of midwifery), there is even less recognition of the very existence of a field of "breastfeeding expertise". Thus it is hardly surprising, if no less distressing, that research and policy-making regarding the infant feeding component of mother to child transmission (MTCT) of HIV has taken place internationally with very little regard for the need for input from "breastfeeding experts". This was true from the first WHO Expert Meeting on HIV and Breastfeeding in Geneva in June 1987 which I attended as an observer on behalf of the Swedish International Development Agency-the only other attendee I would have considered to be a breastfeeding expert was another observer, sent by USAID.

At that time, scientifically, we had only a few case studies to go on. Since then, a number of epidemiological and observational studies have been published, and one randomized study. Sadly, nearly all have had serious methodological limitations. Among these limitations has been the failure to use rigorous definitions in infant feeding research. The major exception is one observational study (1) conducted by some of the coauthors of a paper that appears in this issue of Acta Padiatrica by Bland et al. (2). They were the first and, so far, only research team to examine MTCT among exclusively breastfed infants.

"Exclusive breastfeeding" must be carefully defined to fit the needs of the study at hand. Readers beware, as such definitions are usually lacking, and bizarre ones such as "less than $100 \mathrm{ml}$ infant formula per day" were common in the scientific literature until recently. Those who argued for strict definitions were considered to be fanatics initially, especially if they even wanted water to be excluded. A study from the Philippines examined its data in response to a request in preparation for the meeting that established WHO definitions (3). In this study it was found that even non-nutritive supplementation of 2-mo-old breastfed infants led to higher levels of diarrhoea (4). Otherwise, we might not have been given a strict WHO definition.

These WHO-approved breastfeeding definitions were designed for use only with descriptive household surveys. Thus each researcher dealing with breastfeeding in any other context must come up with his or her own definitions. Fortunately, this has been occurring more often in the literature in recent years.

Bland et al. have done a commendable job in this context, following on other recent studies published from Africa in this "era of AIDS" $(5,6)$. Using both longitudinal and cross-sectional data, they have shown how uncommon exclusive breastfeeding is in an area of South Africa. (However, their use of a 48 -h recall period for part of their analysis makes comparison of their data with others problematic.) Interestingly, this seems to have been much shorter than mothers' intentions (according to their own definition of exclusive breastfeeding). Reminiscent of the comparisons made using only longitudinal data by Aarts et al. (7), Bland et al. have attempted to compare immediate recall with recall from birth, and found that their long-term recall method appeared to lead to an overestimation of the proportion of mothers who had exclusively breastfed from birth up to various ages.

But even stricter definitions are needed for studies examining associations between infant feeding and infant health. A major problem is that the WHO definitions are based on data from 24-h recalls. This ignores the fact that many infants shift from one infant feeding category to another (8). Prelacteal feeding, in particular, is nearly universal in many parts of the world (9).

For certain purposes, definitions may also need to be even stricter. Can vitamin and mineral supplements have an impact on certain outcomes? Can syrups be contaminated? Can antibiotics, because of their effect on gut microflora, have an impact (10)? Researchers need to think through such issues and not just use the WHO definitions because they are the only "approved" ones around! 


\section{References}

1. Coutsoudis A, Pillay K, Kuhn L, Spooner E, Tsai WY, Coovadia HM. Method of feeding and transmission of HIV-1 from mothers to children by 15 months of age: prospective cohort study from Durban, South Africa. AIDS 2001; 15: 379-7

2. Bland R, Rollins NC, Coutsoudis A, Coovadia HM, for the Child Health Group. Breastfeeding practices in an area of high HIV prevalence in rural South Africa. Acta Paediatr 2002; 91: 704-12

3. WHO Division of Child Health and Development. Indicators for assessing breastfeeding practices. Geneva: WHO; 1991. Report No. WHO/CDD/SER/91.14

4. Popkin B, Yamamoto M, Griffin C. Breastfeeding and diarrhea morbidity. Pediatrics 1990; 86: 874-82

5. Shirima R, Greiner T, Kylberg E, Gebre-Medhin M. Exclusive breast-feeding is rarely practised in rural and urban Morogoro, Tanzania. Public Health Nutr 2001; 4: 147-54

6. de Paoli M, Manongi R, Helsing E, Klepp K-I. Exclusive breastfeeding in the era of AIDS. J Hum Lactation 2001; 17: $313-20$
7. Aarts C, Kylberg E, Hornell A, Hofvander Y, Gebre-Medhin M, Greiner T. How exclusive is exclusive breastfeeding? A comparison of data since birth with current status data. Int $\mathrm{J}$ Epidemiol 2000; 29: 1041-6

8. Zohoori N, Popkin BM, Fernandez ME. Breast-feeding patterns in the Philippines: a prospective analysis. J Biosoc Sci 1993; 25: $127-38$

9. Morse JM, Jehle C, Gamble D. Initiating breastfeeding: a world survey of the timing of postpartum breastfeeding. Int J Nurs Stud 1990; 27: 303-13

10. Bennet R, Eriksson M, Nord C-F, Zetterström R. Fecal bacterial microflora of newborn infants during intensive care management and treatment with five antiobiotic regimens. Pediatr Infect Dis 1986; 5: 533-39

Ted Greiner, International Nutrition Research Group, Department of Women's and Children's Health, Uppsala Academic Hospital, Entrance 11, SE-751 85 Uppsala, Sweden (Tel. +46 186115 937, fax.+4618508013,e-mail.ted_greiner@hotmail.com) 